\title{
Electrically Tunable Hot-Silicon Terahertz Attenuator
}

\author{
Minjie Wang, ${ }^{1}$ Robert Vajtai, ${ }^{2}$ Pulickel M. Ajayan, ${ }^{2}$ and Junichiro Kono ${ }^{1,2,3}$, a) \\ ${ }^{1)}$ Department of Electrical and Computer Engineering, Rice University, Houston, Texas 7ro05, \\ $U S A$ \\ ${ }^{2)}$ Department of Materials Science and NanoEngineering, Rice University, Houston, Texas 77005, \\ USA \\ ${ }^{3)}$ Department of Physics and Astronomy, Rice University, Houston, Texas 77005, \\ $U S A$
}

(Dated: 10 September 2014)

\begin{abstract}
We have developed a continuously tunable, broadband terahertz attenuator with a transmission tuning range greater than $10^{3}$. Attenuation tuning is achieved electrically, by simply changing the DC voltage applied to a heating wire attached to a bulk silicon wafer, which controls its temperature between room temperature and $\sim 550 \mathrm{~K}$, with the corresponding free-carrier density adjusted between $\sim 10^{11} \mathrm{~cm}^{-3}$ and $\sim 10^{17} \mathrm{~cm}^{-3}$. This 'hot-silicon'-based terahertz attenuator works most effectively at 450-550 K (corresponding to a DC voltage variation of only $\sim 7 \mathrm{~V}$ ) and completely shields terahertz radiation above $550 \mathrm{~K}$ in a frequency range of $0.1-$ 2.5 THz. Both intrinsic and doped silicon wafers were tested and demonstrated to work well as a continuously tunable attenuator. All behaviors can be understood quantitatively via the free-carrier Drude model taking into account thermally activated intrinsic carriers.
\end{abstract}

PACS numbers: Valid PACS appear here

Keywords: terahertz, attenuation, shielding, hot silicon

Terahertz $(\mathrm{THz})$ technologies ${ }^{1-7}$ are rapidly advancing, opening up diverse, new opportunities, including wireless communications, ${ }^{8}$ nonlinear optical spectroscopy, ${ }^{9}$ and biomedical optics. ${ }^{10}$ Much progress has been made in recent years in finding novel ways to generate, ${ }^{11}$ detect, ${ }^{12,13}$ and manipulate ${ }^{14} \mathrm{THz}$ waves. However, compared to other spectral ranges, there still remains much room for developing more compact and versatile devices for polarizing, filtering, and modulating $\mathrm{THz}$ waves. ${ }^{15-18}$

Attenuating $\mathrm{THz}$ radiation precisely to the desired intensity is an important requirement in spectroscopy, imaging, and communications. However, commercial attenuators are available only in discrete manners, using metalized films with different thicknesses. A continuously controllable $\mathrm{THz}$ attenuator based on optically excited quantum wells was demonstrated, but the amount of change in transmission was only $60 \%$; $^{19}$ a similar device based on optically excited silicon was demonstrated to have an extinction ratio of $10 \mathrm{~dB}$, but it was a narrowband attenuator. ${ }^{20}$ Humidity controlled attenuation has been suggested, but atmospheric attenuation is not spectrally flat, having only several $\sim 0.2 \mathrm{THz}$-wide windows to use for attenuation and thus distorting $\mathrm{THz}$ waveforms; also, absorption of air with relative humidity up to $70 \%$ can only absorb $\sim 30 \% \mathrm{THz}$ signal in these windows. Furthermore, a theoretical proposal for $\mathrm{THz}$ attenuation through rain has been made, ${ }^{21}$ but there has been no experimental demonstration.

Here, we report on the development of an electrically continuously controllable, broadband $\mathrm{THz}$ attenuator based on silicon, with a spectrally fat response preserving the waveforms of incident $\mathrm{THz}$ radiation with no

\footnotetext{
a)Electronic mail: kono@rice.edu.
}

particular polarization dependence. The attenuation ratio can be made larger than $10^{3}$, which is achieved by changing the DC voltage applied to a heating wire attached to a bulk silicon wafer; the heater increases the temperature of silicon from $300 \mathrm{~K}$ to $\sim 550 \mathrm{~K}$, increasing the free-carrier density from $\sim 10^{11} \mathrm{~cm}^{-3}$ to $\sim 10^{17} \mathrm{~cm}^{-3}$. Above $550 \mathrm{~K}$, any incident $\mathrm{THz}$ radiation is blocked in a frequency range of $0.1-2.5 \mathrm{THz}$. Either an intrinsic or doped silicon wafer works as a continuously tunable attenuator. All behaviors can be understood by analyzing free-carrier absorption of $\mathrm{THz}$ radiation by taking into consideration the temperature dependence of carrier density and mobility within the Drude model.

Figure 1(a) shows a schematic diagram of the fabricated device structure, together with the experimental geometry employed. We used two types of silicon wafers: i) a 1-mm-thick intrinsic silicon wafer (resistivity $=5000$ $10000 \Omega$-cm) without any $\mathrm{SiO}_{2}$ layer and ii) a $440-\mu \mathrm{m}-$ thick lightly $p$-doped silicon wafer (resistivity 5 -10 $\Omega$-cm) capped with a 300-nm-thick $\mathrm{SiO}_{2}$ layer. A 40-nm-thick layer of $\mathrm{Pt}$ was electron-beam deposited on the sample through a laser-cut shadow mask, leaving a window with a diameter of $5 \mathrm{~mm}$ for the $\mathrm{THz}$ wave to go through. The Pt layer worked as a resistive heater to heat up the sample with a DC current supplied from a power source. The temperature was measured with a temperature sensor placed at the edge of the $\mathrm{THz}$ window. The temperature-voltage conversion is shown in Figure 1(b). For the intrinsic silicon wafer, a 100 -nm-thick $\mathrm{Al}_{2} \mathrm{O}_{3}$ layer was electron-beam deposited before depositing the $\mathrm{Pt}$ layer, to avoid shorting the resistor when the temperature went high and the resistance of silicon wafer decreased significantly. A typical $\mathrm{THz}$ time-domain spectroscopy system was used for generating and detecting coherent $\mathrm{THz}$ waves. The $\mathrm{THz}$ wave was normal incident onto 

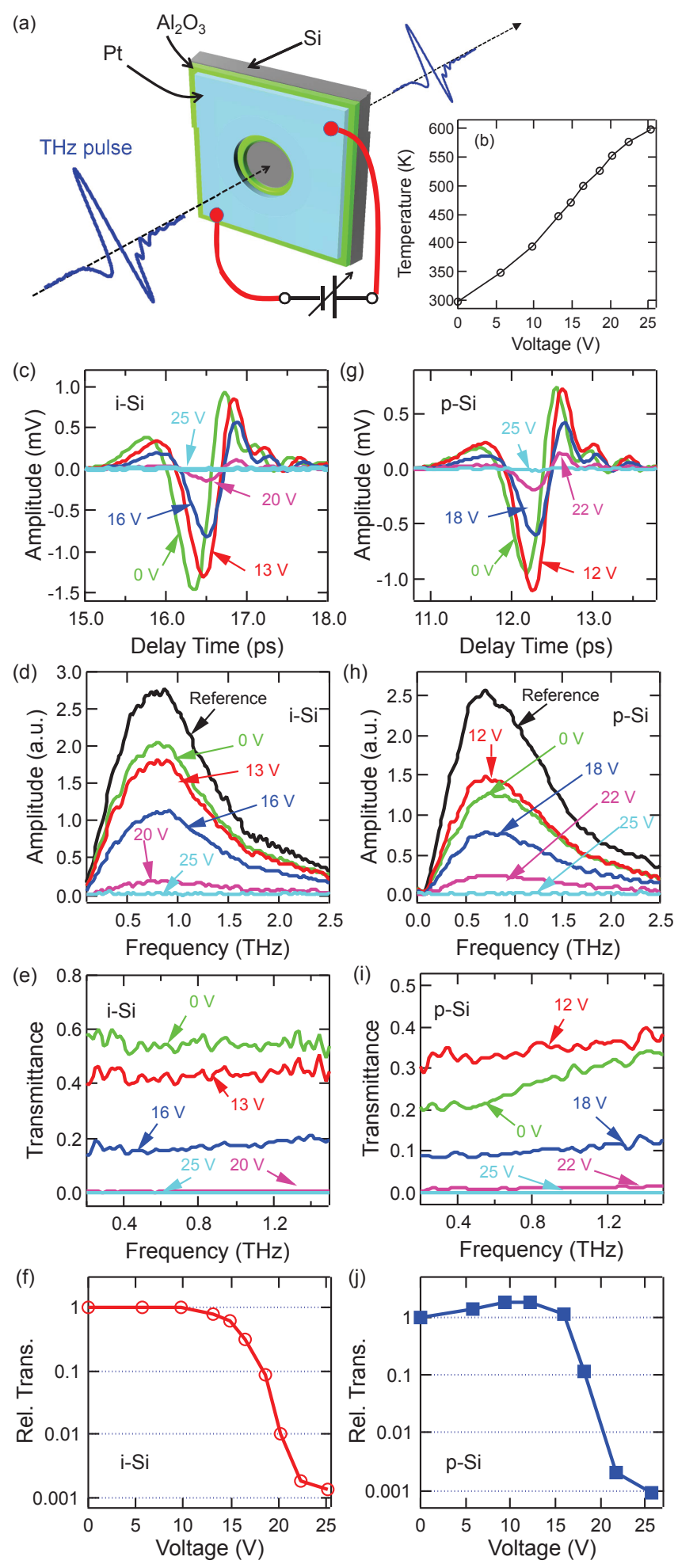

FIG. 1. (a) Experimental configuration and device structure. (b) Conversion between the voltage applied to the heater and the sample temperature. Time-domain waveforms of $\mathrm{THz}$ radiation transmitted through (c) intrinsic Si and (d) p-doped $\mathrm{Si}$ at various applied voltages. Amplitude spectra of transmitted $\mathrm{THz}$ radiation for (e) intrinsic $\mathrm{Si}$ and (f) $p$-doped $\mathrm{Si}$ at various applied voltages. Transmittance spectra for $(\mathrm{g})$ intrinsic Si and (h) $p$-doped Si at various applied voltages. Voltage dependence of transmission normalized to the zero-voltage transmission for (i) intrinsic Si and (j) $p$-doped Si. the sample, and the transmitted wave was detected and recorded as a function of voltage applied to the Pt layer.

Figure 1(c) shows time-domain waveforms of $\mathrm{THz}$ radiation transmitted through the intrinsic silicon device at several applied voltages. As the voltage increases, $\mathrm{THz}$ transmission decreases dramatically. Corresponding frequency-domain data for the sample and reference $\left(\mathrm{N}_{2}\right)$ were obtained through Fourier transform of the timedomain data above, and the resulting amplitude spectra are shown in Figure 1(d). Figure 1(e) shows power transmittance spectra calculated from the $\mathrm{THz}$ amplitude spectra, defined as $T(\omega)=\left|\tilde{E}_{s}(\omega) / \tilde{E}_{r}(\omega)\right|^{2}$, where $\tilde{E}_{s}(\omega)$ and $\tilde{E}_{r}(\omega)$ are the complex $\mathrm{THz}$ amplitudes in the frequency domain for the sample and reference, respectively. At room temperature, the transmittance is $\sim 0.55$ in the whole frequency range. The transmittance starts dropping rapidly above $13 \mathrm{~V}$ and decreases below our detectability limit above $25 \mathrm{~V}$; this trend is more clearly seen in Figure 1(f), which plots the transmitted $\mathrm{THz}$ power, normalized to the room temperature value, as a function of voltage.

The same measurements were performed for the $p$ doped silicon device, and the results are shown in Figures $1(\mathrm{~g}),(\mathrm{h}),(\mathrm{i})$, and $(\mathrm{j})$. The results are similar to those for the undoped silicon device, but there are two conspicuous differences. First, at room temperature, there is stronger absorption than the intrinsic silicon device, especially at low frequencies. This is consistent with the expectation that free carriers in the doped sample absorb more low-frequency $\mathrm{THz}$ radiation through Drude absorption; see Equation (3). Second, before the drop of transmission occurs at high voltages, the transmission initially increases slightly, as observed in Figures 1(h) and $1(\mathrm{i})$, reaching a maximum around $11-13 \mathrm{~V}$, as shown in Figure 1(j).

For each applied voltage (i.e., temperature, $T$ ), we performed data analysis to extract the complex refractive index, $\tilde{n}(\omega) \equiv n(\omega)-i \kappa(\omega)$. When the THz wave is normal incident to a sample with thickness $d, \tilde{E}_{s}(\omega) / \tilde{E}_{r}(\omega)$ can be expressed as

$$
\frac{\tilde{E}_{s}(\omega)}{\tilde{E}_{r}(\omega)}=\frac{4 \tilde{n}(\omega)}{\{\tilde{n}(\omega)+1\}^{2}} \exp \left[-i \frac{\omega d}{c}\{\tilde{n}(\omega)-1\}\right] .
$$

We applied this formula to the data shown in Figure 1 to determine $\tilde{n}(\omega)$ for each $T$. Furthermore, we determined the complex dielectric constant, $\tilde{\varepsilon}(\omega)$, and complex conductivity, $\tilde{\sigma}(\omega)$, through

$$
\tilde{n}^{2}(\omega)=\tilde{\varepsilon}(\omega)=\varepsilon_{\mathrm{Si}}-i \frac{\tilde{\sigma}(\omega)}{\omega \varepsilon_{0}}
$$

where $\varepsilon_{\mathrm{Si}}=11.6$ is the relative dielectric constant of intrinsic silicon and $\varepsilon_{0}$ is the permittivity of free space.

The real part of the complex conductivity is shown in Figures 2(a) and 2(b) for the intrinsic Si and $p$-doped $\mathrm{Si}$, respectively, at different temperatures. Finally, from the imaginary part of the refractive index, $\kappa(\omega)$, we obtained the absorption coefficient, $\alpha(\omega)$, through $\alpha(\omega)=$ 

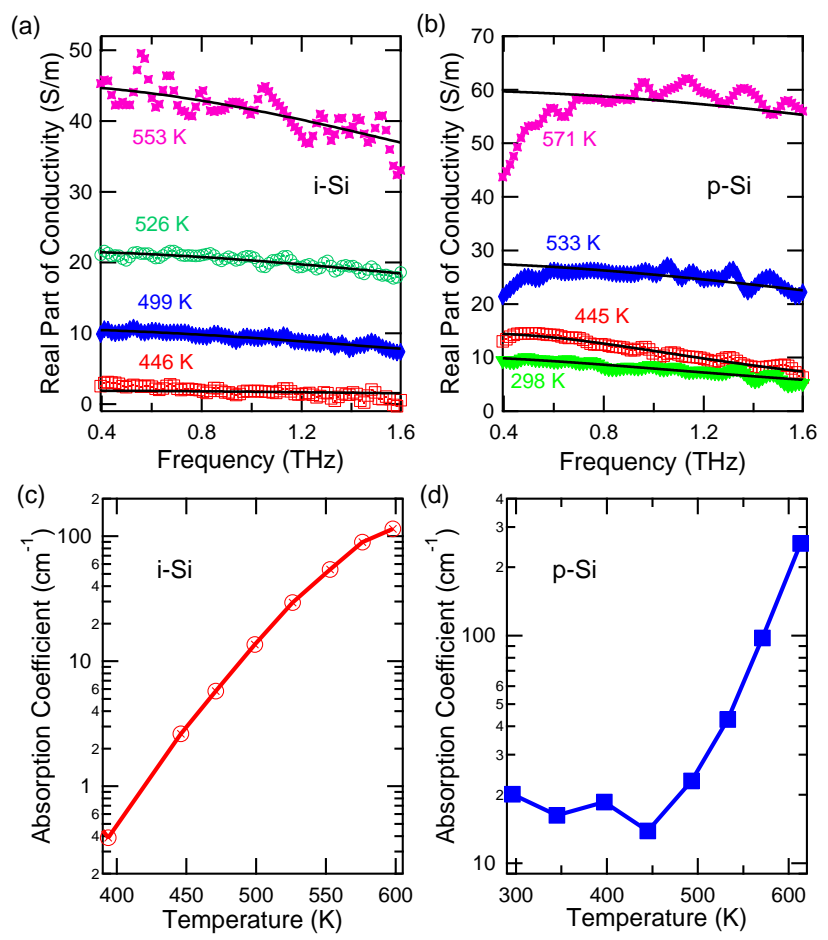

FIG. 2. Extracted optical conductivity spectra for the (a) intrinsic and (b) p-doped silicon devices at different temperatures, together with Drude fits (solid lines). Extracted temperature dependence of absorption coefficient for the (a) intrinsic and (b) $p$-doped silicon devices. In the case of intrinsic silicon, the absorption coefficient changes by three orders of magnitude as the temperature changes from 400 to $600 \mathrm{~K}$.

$2 \omega \kappa(\omega) / c$ (data not shown). We spectrally integrated $\alpha(\omega)$ in the whole frequency region at each $T$ and plotted its values versus $T$ in Figures 2(c) and 2(d). In the case of intrinsic silicon, the absorption coefficient monotonically increases by three orders of magnitude as $T$ changes from $\sim 400$ to $\sim 600 \mathrm{~K}$. In the case of $p$-doped silicon, the absorption coefficient initially decreases with increasing $T$ but increases dramatically above $\sim 450 \mathrm{~K}$. All these experimental data can be fitted with the Drude model,

$$
\tilde{\sigma}(\omega)=\frac{\sigma_{0}}{1-i \omega \tau},
$$

where $\sigma_{0}$ is the DC conductivity and $\tau$ is the carrier scattering time. The Drude model fitting results for the real part of the complex conductivity are also shown as solid lines in Figures 2(a) and 2(b), which allowed us to obtain the $\sigma_{0}$ and $\tau$ values at different $T$ for both types of silicon devices. The temperature dependence of the extracted parameters, i.e., DC conductivity, $\sigma_{0}$, carrier mobility, $\mu$, and carrier density, $n$, is shown in Figure 3.

Figure 3(a) shows that in intrinsic silicon $\sigma_{0}$ increases monotonically with increasing $T$ while in the $p$-doped silicon sample $\sigma_{0}$ exhibits a slight initial decrease. For the intrinsic silicon device, since the carrier density is too small at low temperatures, Drude-model fitting was
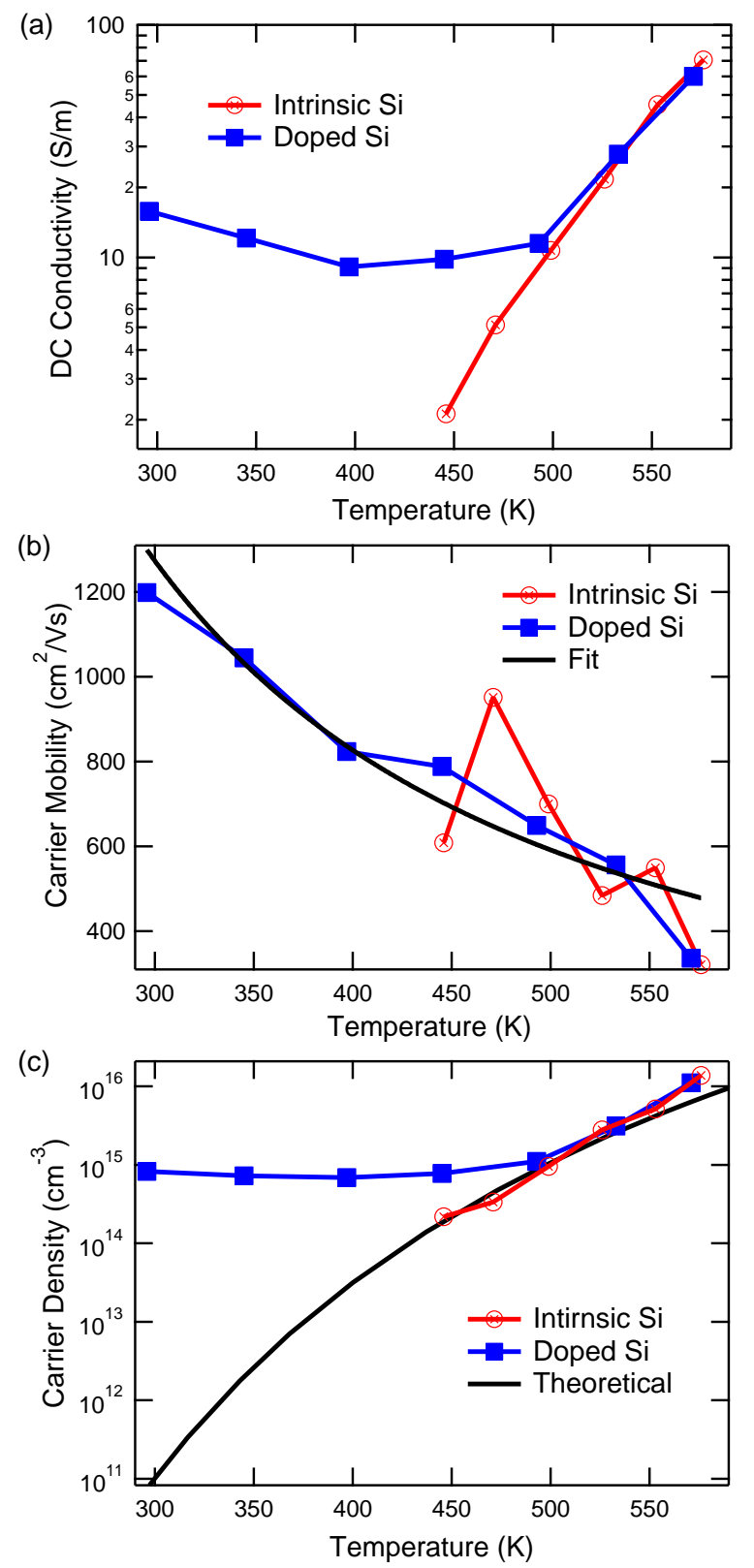

FIG. 3. (a) DC conductivity, $\sigma_{0}$, (b) carrier mobility, $\mu$, and (c) carrier density, $n$, versus temperature extracted through Drude fits to data in Figure 2(a) and 2(b) for the intrinsic and $p$-doped silicon devices, respectively. The black solid line in (b) is a fit through $\mu=A \cdot T^{-3 / 2}$, as expected from acoustic phonon scattering. The black solid line in (c) is the theoretical curve representing Equation (4).

not applicable, and thus, we ignored the first three sets of data. The obtained $\sigma_{0}$ value for the $p$-doped silicon sample at room temperature is $\sigma_{0}=15.8 \mathrm{~S} / \mathrm{m}$, which agrees well with the data provided by the vendor (10$20 \mathrm{~S} / \mathrm{m}$ ) for this $p$-doped silicon wafer.

The mobility, $\mu$, of carriers can be derived from the scattering time $\tau$ through $\mu=e \tau / m^{*}$, where $m^{*}=$ 
$1 /\left[\left(1 / m_{e}^{*}\right)+\left(1 / m_{h}^{*}\right)\right]$ is the reduced effective mass of electrons and holes in silicon, $m_{e}^{*}=0.26 m_{0}$ is the electron effective mass, $m_{h}^{*}=0.36 m_{0}$ is the hole effective mass, and $m_{0}$ is the electron mass in vacuum. As shown in Figure 3(b), the two types of silicon samples show the same type of decrease in $\mu$ with increasing $T$. This is understandable in terms of carrier-phonon interaction. As $T$ increases, the carriers are more likely to be scattered by the thermally activated phonons, which lowers $\mu$. It is known that the carrier mobility in silicon is proportional to $T^{-3 / 2}$ due to acoustic phonon scattering. ${ }^{22}$ The black solid line in Figure 3(b) shows a fitting curve with $\mu=$ $A \cdot T^{-3 / 2}$, and we can see that it agrees very well with the experimental data.

Finally, the carrier density, $n$, can be derived from the relation $\sigma_{0}=e \mu n$. The intrinsic carrier density, $n_{i}$, in silicon at temperature $T[\mathrm{~K}]$ is given by

$$
\begin{aligned}
n_{i} & =\left(N_{c} N_{v}\right)^{1 / 2} \exp \left(-\frac{E_{g}}{2 k_{\mathrm{B}} T}\right) \\
& =1.69 \times 10^{26}\left(\frac{T}{300}\right)^{1 / 2} e^{-6370 / T}\left[\mathrm{~cm}^{-3}\right]
\end{aligned}
$$

where $N_{c}$ and $N_{v}$ are the effective density of states for electrons and holes, respectively, $E_{g}=1.12 \mathrm{eV}$ is the room-temperature band gap of silicon, and $k_{\mathrm{B}}$ is the Boltzmann constant. The black solid curve represents Equation (4) without any adjustable parameter, which agrees with the obtained experimental data well.

Overall, the data for the intrinsic silicon device an be interpreted as a consequence of intrinsic carriers thermally activated across the band gap. For the $p$-doped silicon sample, extra carriers were obser $\&$ at lower temperatures, which should be due to the doped (extrinsic) carriers. However, the intrinsic carrier density also increases dramatically with temperature and starts to dominate the whole carrier density even in the $p$-doped silicon device above $\sim 500 \mathrm{~K}$, making it similar to the intrinsic silicon device. The dramatic increase in carrier density occurs in the temperature range where the $\mathrm{THz}$ transmission drops. This further leads us to conclude that the $\mathrm{THz}$ transmission drop we observed is caused by the thermally activated carriers across the band gap. In addition, as the temperature increases, the carrier mobility decreases, which results in the initial increase in transmission observed in the doped silicon device.

In conclusion, we fabricated and characterized a novel $\mathrm{THz}$ attenuator whose transmission can be continuously tuned by three orders of magnitude. The operation principle of the device is based on thermal activation of carriers across the bad gap in silicon, which is achieved through a heating wire attached. The temperature was adjusted between $300 \mathrm{~K}$ and $\sim 550 \mathrm{~K}$, which re- sulted in dramatic free-carrier density control between $\sim 10^{11} \mathrm{~cm}^{-3}$ and $\sim 10^{17} \mathrm{~cm}^{-3}$. Both intrinsic and doped silicon wafers work under the same mechanism, but the effect is more drastic in the intrinsic silicon device due to the absence of extrinsic carriers at room temperature. All the observations were accurately reproduced through the free-carrier Drude model. The developed device can be useful for diverse applications in $\mathrm{THz}$ spectroscopy, imaging, and communications.

\section{ACKNOWLEDGMENTS}

This work was supported by the National Science Foundation through Grant No. OISE-0968405.

${ }^{1}$ P. H. Siegel, IEEE Trans. Microwave Theor. Tech. 50, 910 (2002).

${ }^{2}$ B. Ferguson and X.-C. Zhang, Nat. Mater. 1, 26 (2002).

${ }^{3}$ D. M. Mittleman, ed., Sensing with Terahertz Radiation (Springer, Berlin, 2003).

${ }^{4}$ K. Sakai, ed., Terahertz Optoelectronics (Springer, Berlin, 2005).

${ }^{5}$ M. Tonouchi, Nat. Photon. 1, 97 (2007).

${ }^{6}$ D. M. Mittleman, Nat. Photon. 7, 666 (2013).

${ }^{7}$ R. R. Hartmann, J. Kono, and M. E. Portnoi, Nanotechnology 25, 322001 (2014).

${ }^{8}$ T. Nagatsuma, S. Horiguchi, Y. Minamikata, Y. Yoshimizu, S. Hisatake, S. Kuwano, N. Yoshimoto, J. Terada, and H. Takahashi, Opt. Exp. 21, 23736 (2013).

${ }^{9}$ T. Kampfrath, K. Tanaka, and K. A. Nelson, Nat. Photon. 7 , 680 (2013).

${ }^{10}$ L. V. Titova, A. K. Ayesheshim, A. Golubov, D. Fogen, R. Rodriguez-Juarez, F. A. Hegmann, and O. Kovalchuk, Biomed. Opt. Exp. 4, 559 (2013).

${ }^{11}$ K. Vijayraghavan, Y. Jiang, M. Jang, A. Jiang, K. Choutagunta, A. Vizbaras, F. Demmerle, G. Boehm, M. C. Amman, and M. A. Belkin, Nat. Commun. 4, 2021 (2013).

${ }^{12}$ S. Komiyama, IEEE J. Sel. Top. Quant. Elec. 17, 54 (2011).

${ }^{13} \mathrm{X}$. He, N. Fujimura, J. M. Lloyd, K. J. Erickson, A. A. Talin, Q. Zhang, W. Gao, Q. Jiang, Y. Kawano, R. H. Hauge, F. Léonard, and J. Kono, Nano Lett. 14, 3953 (2014).

${ }^{14}$ H.-T. Chen, W. J. Padilla, J. M. Zide, A. C. Gossard, A. J. Taylor, and R. D. Averitt, Nature 444, 597 (2006).

${ }^{15}$ P. Kužel and F. Kadlec, C. R. Physique 9, 197 (2008).

${ }^{16}$ M. Rahm, J.-S. Li, and W. J. Padilla, J. Infrared Milli. Terahz Waves 34, 1 (2013).

${ }^{17}$ L. Ren, C. L. Pint, T. Arikawa, K. Takeya, I. Kawayama, M. Tonouchi, R. H. Hauge, and J. Kono, Nano Lett. 12, 787 (2012).

${ }^{18}$ L. Ren, Q. Zhang, J. Yao, Z. Sun, R. Kaneko, Z. Yan, S. Nanot, Z. Jin, I. Kawayama, M. Tonouchi, J. M. Tour, and J. Kono, Nano Lett. 12, 3711 (2012).

${ }^{19}$ N. E. Hecker, I. H. Libofl, M. Hempel, S. Baumgartner, M. KoCha, P. Dawson, and J. Feidmann, Proc. SPIE 3828, 378 (1999).

${ }^{20}$ J. S. Li and J. Q. Yao, Microwave Opt. Technol. Lett. 50, 1810 (2008).

${ }^{21}$ Y. Luo, W. X. Huang, and Z. Y. Luo, Optoelectron. Lett. 8, 310 (2012).

${ }^{22}$ P. Yu, Fundamentals of Semiconductors: Physics and Materials Properties, 4th ed. (Springer, Berlin, 2010). 
(a)

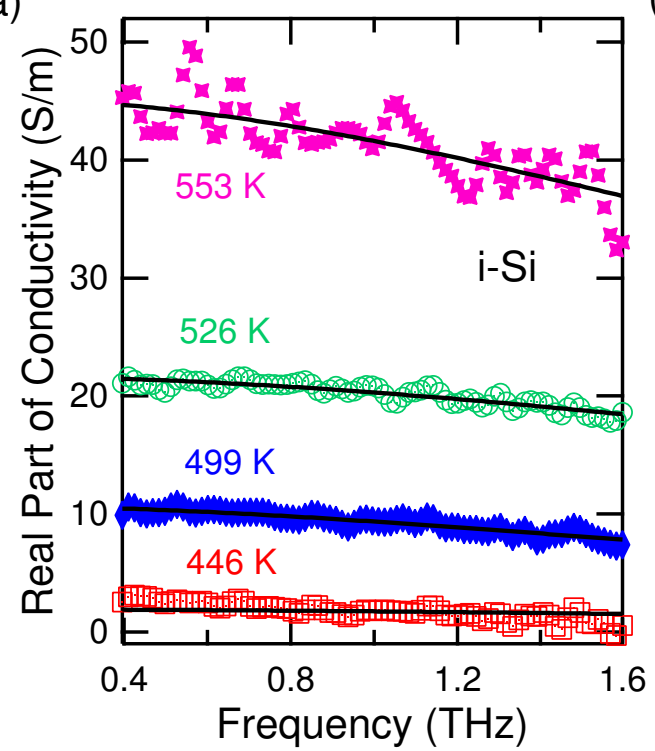

(c)

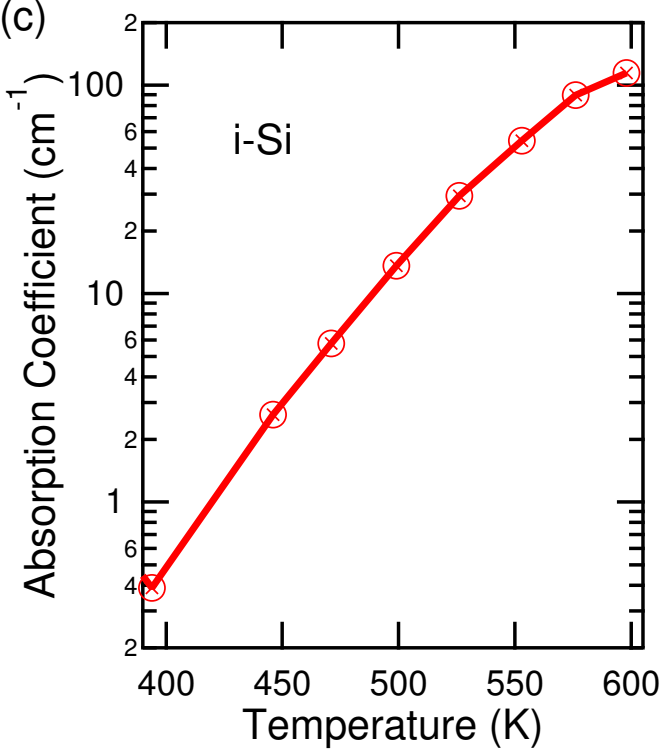

(b)
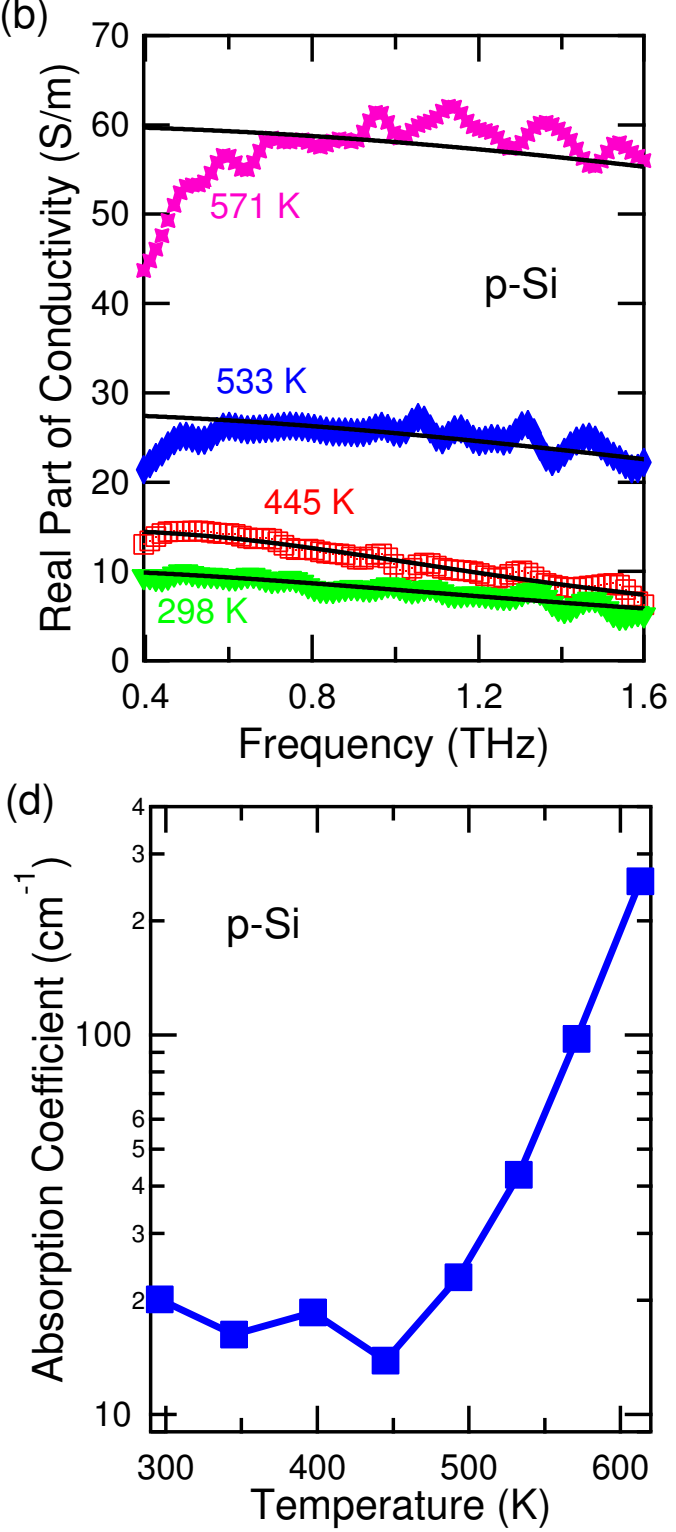

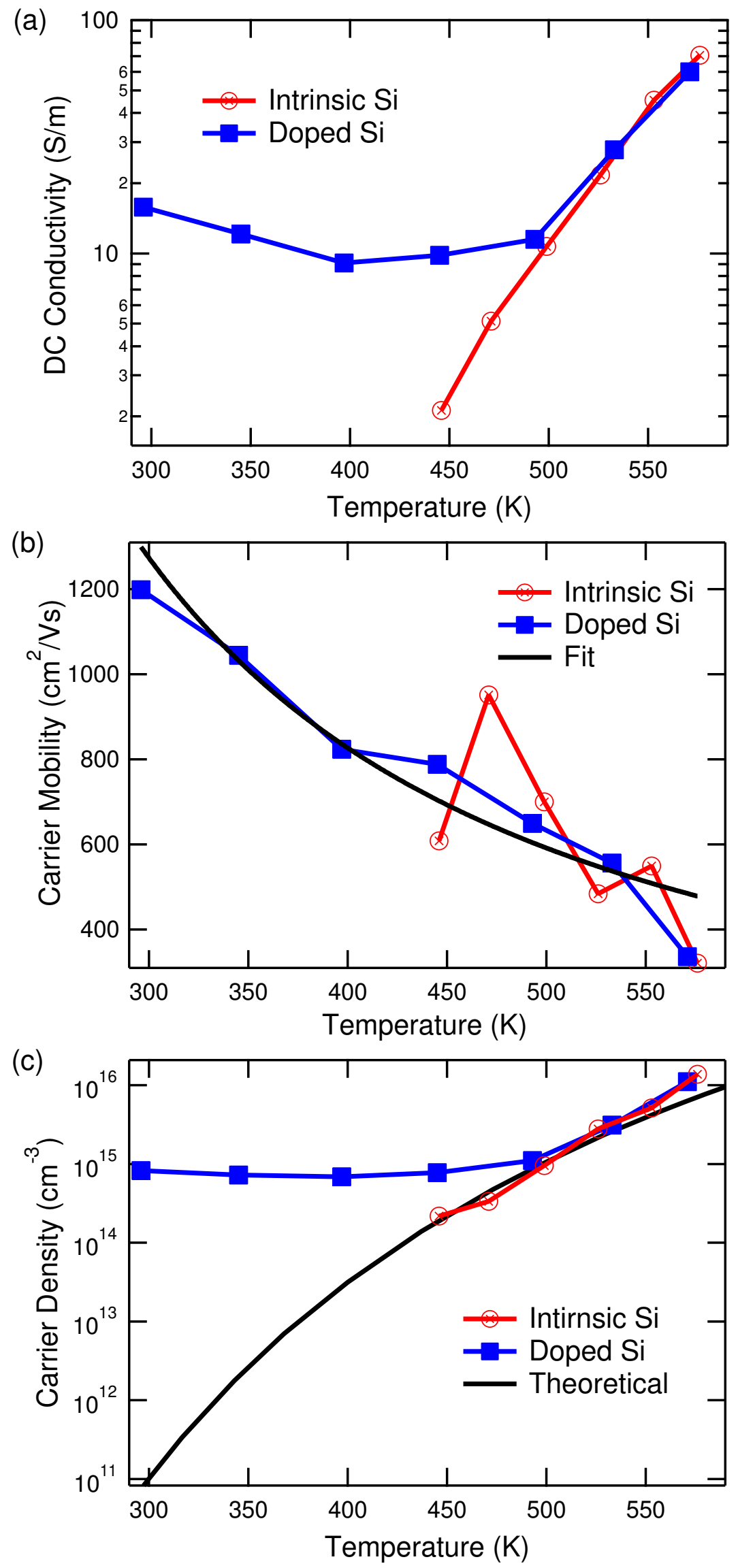